\title{
An Algebraic Framework for Quadratic Invariance
}

\author{
Laurent Lessard $^{1} \quad$ Sanjay Lall ${ }^{2}$ \\ IEEE Conference on Decision and Control, pp. 2698-2703, 2010
}

\begin{abstract}
In this paper, we present a general algebraic framework for analysing decentralized control systems. We consider systems defined by linear fractional functions over a commutative ring. This provides a general algebraic formulation and proof of the main results of quadratic invariance, as well as naturally covering rational multivariable systems, systems with delays, and multidimensional systems. The approach extends to the extended class of internally quadratically invariant systems.
\end{abstract}

\section{Introduction}

In decentralized control, multiple controllers are used to control a plant with many inputs and outputs. Each controller has access to different outputs (measurements), and controls a different subset of the inputs. Such systems arise when the plant is itself distributed, and it would be infeasible or otherwise impossible to control it using a centralized computer.

In the linear fractional transformation (LFT) formulation, the closed-loop map is given by

$$
f(P, K)=P_{11}+P_{12} K\left(I-P_{22} K\right)^{-1} P_{21}
$$

where the $P_{i j}$ are system parameters. We seek a controller $K$ that minimizes a performance norm

$$
\begin{aligned}
\operatorname{minimize} & \|f(P, K)\| \\
\text { subject to } & K \in S
\end{aligned}
$$

where $S$ is the set of admissible controllers.

For a general constraint sets $S$, finding the optimal controller is a hard problem. Indeed, the set $f(P, S)$ need not be convex, and the optimal controller need not be linear [11]. However, decentralized problems with linear optimal controllers have also been identified. The largest such class known is defined by a property called quadratic invariance [8, 9].

Henceforth, we will use the abbreviation $G=P_{22}$. Quadratic invariance is a simple algebraic condition. We

\footnotetext{
${ }^{1}$ L. Lessard is with the Department of Aeronautics and Astronautics at Stanford University, Stanford, CA 94305, USA. lessard@stanford.edu

${ }^{2} \mathrm{~S}$. Lall is with the Department of Electrical Engineering and Aeronautics and Astronautics at Stanford University, Stanford, CA 94305, USA. lall@stanford.edu

${ }^{3}$ This work was supported by NSF grant number 0642839
}

say $S$ is quadratically invariant (QI) with respect to $G$ if

$$
K G K \in S \quad \text { for every } K \in S \text {. }
$$

Roughly speaking, if this condition holds and $S$ is a subspace, the set $f(P, S)$ is affine, and the controller synthesis problem can be posed as a convex optimization problem and thereby solved.

This result holds in a very broad sense. Subject to some technical conditions, it holds when the plant and controllers are bounded linear operators from one Banach space to another [8]. More generally, it holds for causal maps on extended spaces [9]. This encompasses continuous and discrete systems, stable or unstable, and even systems with delays.

In both cases, the results are proven using tools from analysis. Since the maps in question are potentially infinite-dimensional, questions of convergence arise. One must also take care in defining appropriate topologies so that the notion of convergence is the appropriate one. Both results require $S$ to be a closed subspace, which is problematic when we seek controllers expressible as rational transfer functions.

Given that the QI result holds in a very broad sense, and that the QI condition is algebraic in nature, it encourages one to seek an algebraic framework in which the results can be expressed naturally. In this paper, we present such a framework. We consider plants and controllers to be matrices whose entries belong to a commutative ring. A similar framework was suggested [10], which generalizes the notion of a transfer function matrix, and applies it to feedback stabilization.

In Section 2, we cover some preliminaries; results that hold in the general ring case. In Section 3, we consider a more specific ring; multidimensional rational functions. We prove both QI and internal quadratic invariance [4] results in this case. Finally, in Section 4, we discuss some applications.

\section{Commutative Rings}

A commutative ring is a tuple $(R,+, \cdot)$ consisting of a set $R$, and two binary operations which we call addition and multiplication, respectively. The following properties hold for all $a, b, c \in R$. First, $(R,+)$ is an abelian group:
i) Closure: $a+b \in R$
ii) Commutativity: $a+b=b+a$ 
iii) Associativity: $a+(b+c)=(a+b)+c$

iv) Additive identity: there exists an element $0_{R} \in R$ such that $a+0_{R}=0_{R}+a=a$

v) Additive inverse: there exists an element $-a \in R$ such that $a+(-a)=(-a)+a=0_{R}$

Next, $(R, \cdot)$ is a commutative monoid:

vi) Closure: $a \cdot b \in R$

vii) Commutativity: $a \cdot b=b \cdot a$

viii) Associativity: $a \cdot(b \cdot c)=(a \cdot b) \cdot c$

ix) Multiplicative identity: there exists an element $1_{R} \in$ $R$ such that $a \cdot 1_{R}=1_{R} \cdot a=a$

Finally, the addition and multiplication operations satisfy two distributive properties:

x) $a \cdot(b+c)=a \cdot b+a \cdot c$

xi) $(a+b) \cdot c=a \cdot c+b \cdot c$

We will often omit the multiplication symbol, and simply concatenate the variables. So $a b$ should be interpreted as $a \cdot b$. Throughout this paper, we will use $R$ to denote an arbitrary commutative ring satisfying the axioms above.

If some element $a \in R$ has a multiplicative inverse in $R, a$ is called a unit. The set of all units of $R$ forms a group under multiplication, and is denoted $U(R)$. If $U(R)=R \backslash\left\{0_{R}\right\}$, then $R$ is a field.

We will often arrange elements of $R$ into a matrix, and specify dimensions as a superscript. For example, $R^{m \times n}$ denotes the set of $m \times n$ matrices where each entry is an element of $R$.

Some real matrix concepts extend naturally to matrices over $R$. The most basic are matrix addition and matrix multiplication. The determinant det $: R^{n \times n} \rightarrow R$ is well defined, since for any $A \in R^{n \times n}, \operatorname{det}(A)$ is a polynomial in the entries $A_{i j} \in R$. The classical adjoint adj : $R^{n \times n} \rightarrow R^{n \times n}$ also makes sense, because its definition is in terms of determinants of submatrices. For any $A \in R^{n \times n}$, the fundamental property of adjoints extends to the commutative ring case

$$
A \operatorname{adj}(A)=\operatorname{adj}(A) A=\operatorname{det}(A) I_{R} .
$$

where the matrix $I_{R}$ is the identity matrix in $R^{n \times n}$. That is, the matrix whose diagonal and off-diagonal entries are $1_{R}$ and $0_{R}$, respectively. We will use $0_{R}^{n \times n}$ to denote the $n \times n$ matrix whose entries are all $0_{R}$.

The characteristic polynomial of a matrix $A \in$ $R^{n \times n}$ is the function $p_{A}: R \rightarrow R$ defined by $p_{A}(x)=$ $\operatorname{det}\left(A-x I_{R}\right)$. In general, $p_{A}$ is a polynomial of degree $n$

$$
p_{A}(x)=p_{0}+p_{1} x+\cdots+p_{n} x^{n}
$$

where $p_{i} \in R$.
We will also use a notion that generalizes that of a subspace. An $R$-module consists of an abelian group $(H,+)$ and an operation $R \times H \rightarrow H$ (called scalar multiplication), such that for all $r, s \in R$ and $x, y \in H$,
i) $r(x+y)=r x+r y$
ii) $(r+s) x=r x+s x$
iii) $(r s) x=r(s x)$
iv) $1_{R} x=x$

In particular, a subset $S \subset R^{n \times m}$ is an $R$-module if it is closed under addition and satisfies the property $r X \in S$ for all $X \in S$ and $r \in R$.

The most important fact about commutative rings used in this paper is the Cayley-Hamilton theorem. This well-known result for real matrices also holds in $R^{n \times n}$.

Lemma 1. (Cayley-Hamilton) Suppose $A \in R^{n \times n}$. Define the function $\tilde{p}_{A}: R^{n \times n} \rightarrow R^{n \times n}$ as

$$
\tilde{p}_{A}(X)=p_{0} I_{R}+p_{1} X+\cdots+p_{n} X^{n},
$$

where $p_{i}$ are the coefficients of the characteristic polynomial $p_{A}(x)$. Then $\tilde{p}_{A}(A)=0_{R}^{n \times n}$. In other words, $A$ satisfies its own characteristic polynomial.

Proof. See for example, [5, p. 7-8].

The concept of a matrix inverse can also be extended to matrices over $R$. A matrix $A \in R^{n \times n}$ is invertible if $\operatorname{det}(A) \in U(R)$. In this case, the inverse is unique, and equal to

$$
A^{-1}=(\operatorname{det}(A))^{-1} \operatorname{adj}(A)
$$

We can use the Cayley-Hamilton to express the adjoint and hence the inverse as finite sums as well.

Lemma 2. Suppose $A \in R^{n \times n}$ is invertible. There exist $p_{1}, \ldots, p_{n} \in R$ such that

$$
-\operatorname{adj}(A)=p_{1} I_{R}+p_{2} A+\cdots+p_{n} A^{n-1}
$$

Proof. Using Lemma 1, we know that

$$
p_{0} I_{R}+p_{1} A+\cdots+p_{n} A^{n}=0_{R}^{n \times n}
$$

where the $p_{i}$ satisfy $\operatorname{det}\left(A-x I_{R}\right)=p_{0}+p_{1} x+\ldots p_{n} x^{n}$. Setting $x=0_{R}$, we have $p_{0}=\operatorname{det}(A)$. Multiply by $\operatorname{adj}(A)$ on the right, and obtain

$$
\operatorname{det}(A) \operatorname{adj}(A)+p_{1} A \operatorname{adj}(A)+\ldots p_{n} A^{n} \operatorname{adj}(A)=0_{R}^{n \times n}
$$

Now use the fundamental property of the classical adjoint: $\operatorname{adj}(A) A=A \operatorname{adj}(A)=\operatorname{det}(A) I_{R}$.

$$
\operatorname{det}(A)\left(\operatorname{adj}(A)+p_{1} I_{R}+p_{2} A+\cdots+p_{n} A^{n-1}\right)=0_{R}^{n \times n}
$$

Since $A$ is invertible, $\operatorname{det}(A) \in U(R)$ and so $\operatorname{det}(A)$ has a multiplicative inverse. Multiply by this inverse and obtain

$$
-\operatorname{adj}(A)=p_{1} I_{R}+p_{2} A+\cdots+p_{n} A^{n-1},
$$

as required. 


\subsection{Quadratic Invariance for Rings}

In this section, we take the algebraic notion of quadratic invariance [8,9], and show how it fits into the framework of matrices over commutative rings. We begin with the definition of quadratic invariance.

Definition 3. Suppose $G \in R^{m \times n}$ and $S \subset R^{n \times m}$ is an $R$-module. $S$ is quadratically invariant with respect to $G$ if for all $K \in S$, we have $K G K \in S$.

For a particular $G \in R^{m \times n}$, define the set $M \subset R^{n \times m}$ as: $\quad M=\left\{K \in R^{n \times m} \mid\left(I_{R}-G K\right)\right.$ is invertible $\} . \quad \mathrm{A}$ function of interest is $h: M \rightarrow M$ defined as

$$
h(K)=-K\left(I_{R}-G K\right)^{-1}
$$

This notation allows us to succinctly express the closedloop map as $f(P, K)=P_{11}-P_{12} h(K) P_{21}$. Note that the function $h$ has the involutive property, namely $h(h(K))=K$ for every $K \in M$.

Our main approach is to apply the Cayley-Hamilton theorem to show that $h(K)$ can be expressed as a finite sum of terms. When $S$ is quadratically invariant under $G$, each term in the sum belongs to $S$ and so $h(S \cap M)=$ $S \cap M$.

Lemma 4. Suppose $G \in R^{m \times n}$ and $S \subset R^{n \times m}$ is an $R$-module. Further suppose that $2_{R} \in U(R)$. If $S$ is quadratically invariant with respect to $G$, then for all $K \in$ $S$ :

$$
K(G K)^{i} \in S \quad \text { for } i=1,2, \ldots
$$

Proof. The result follows by induction, using the identity:

$$
\begin{aligned}
K(G K)^{i+1}=2_{R}^{-1} & {\left[\left(K+K(G K)^{i}\right) G\left(K+K(G K)^{i}\right)\right.} \\
& \left.-K G K-\left(K(G K)^{i}\right) G\left(K(G K)^{i}\right)\right]
\end{aligned}
$$

where $2_{R}^{-1}$ is the multiplicative inverse of $2_{R}=1_{R}+1_{R}$, which exists by assumption.

Theorem 5. Suppose $G \in R^{m \times n}$ and $S \subset R^{n \times m}$ is an $R$-module. Further suppose that $2_{R} \in U(R)$. If $S$ is quadratically invariant with respect to $G$, then

$$
h(S \cap M)=S \cap M
$$

Proof. Suppose $K \in S \cap M$. Using Lemma 2, write:

$$
\begin{aligned}
h(K) & =-\left(\operatorname{det}\left(I_{R}-G K\right)\right)^{-1} K \operatorname{adj}\left(I_{R}-G K\right) \\
& =\left(\operatorname{det}\left(I_{R}-G K\right)\right)^{-1} \sum_{i=1}^{m} p_{i} K\left(I_{R}-G K\right)^{i-1} \\
& =\sum_{i=1}^{m} h_{i} K(G K)^{i-1}
\end{aligned}
$$

where the $h_{i} \in R$ are obtained by expanding each $\left(I_{R}-G K\right)^{i-1}$ term and collecting like powers of $G K$. All terms in the sum are in $S$, via Lemma 4 . Since $S$ is an $R$-module, it follows that $h(K) \subset S \cap M$. Using the involutive property of $h$, we have $h(S \cap M)=S \cap M$.
Counterexample. We will now show that the requirement $2_{R} \in U(R)$ is necessary. Consider the ring of integers $\mathbb{Z}$, and define:

$$
S=\left\{\left[\begin{array}{ccc}
2 x & y & z \\
y & z & 0 \\
z & 0 & 0
\end{array}\right] \mid x, y, z \in \mathbb{Z}\right\}, \quad G=\left[\begin{array}{lll}
0 & 0 & 0 \\
0 & 0 & 1 \\
0 & 1 & 0
\end{array}\right]
$$

It is easy to check that $S$ is a $\mathbb{Z}$-module, and is quadratically invariant with respect to $G$. Now consider a particular element of $S$ :

$$
K_{0}=\left[\begin{array}{lll}
0 & 0 & 1 \\
0 & 1 & 0 \\
1 & 0 & 0
\end{array}\right]
$$

Note that $\operatorname{det}\left(I-G K_{0}\right)=1$, so $K_{0} \in S \cap M$. However, $h\left(K_{0}\right) \notin S$, so Theorem 5 does not hold. Indeed, $K_{0}\left(G K_{0}\right)^{2} \notin S$, so Lemma 4 does not hold either.

The requirement that $2_{R} \in U(R)$ can be dropped if we strengthen our notion of quadratic invariance. One way to do this is to require that $K_{1} G K_{2} \in S$ for all $K_{1}, K_{2} \in S$.

This result shows that even in a purely algebraic setting, quadratic invariance implies that the set of achievable closed-loop maps is affine. For the remainder of this paper, we will turn our attention to a more specialized commutative ring.

\section{Rational Functions}

For the remainder of this paper, we will work with rational functions of multiple variables. This leads to quadratic invariance results without any closure requirement on $S$. Furthermore, the framework is flexible enough to allow systems with delays or spatiotemporal systems (see Section 4).

Let $\mathbb{R}(\mathbf{s})$ be the set of rational functions in the variables $\mathbf{s}=\left(s_{1}, s_{2}, \ldots, s_{k}\right)$, with coefficients in $\mathbb{R}$. We say that $r \in \mathbb{R}(\mathbf{s})$ is proper if for every $i$, the degree of $s_{i}$ in the numerator is less than or equal to the degree of $s_{i}$ in the denominator. The set of proper rationals will be denoted $\mathbb{R}(\mathbf{s})_{p}$. For example, the rational function

$$
\frac{s_{1} s_{2} s_{3}}{s_{1}^{2}+2 s_{2}+s_{3}}
$$

is proper. Similarly, we define $\mathbb{R}(\mathbf{s})_{s p}$ to be the set of strictly proper rationals. Finally, $\mathbb{R}(\mathbf{s})_{n}$ is the set of "proper but not strictly proper" rationals. That is, each variable $s_{i}$ has the same degree in the numerator and denominator. As a convention, $0 \in \mathbb{R}(\mathbf{s})_{s p}$.

We may alternatively characterize properness by using limits. We state the following lemma without proof.

Lemma 6. Suppose $h \in \mathbb{R}(\mathbf{s})$. For every $i \in\{1, \ldots, k\}$, Let $\bar{s}^{i}=\left\{\bar{s}_{1}, \ldots, \bar{s}_{i-1}, \bar{s}_{i+1}, \ldots, \bar{s}_{k}\right\} \subset \mathbb{R}$ be some assignment of the remaining $k-1$ variables. Define

$$
c_{i}\left(\bar{s}^{i}\right)=\lim _{s_{i} \rightarrow \infty} h\left(\bar{s}_{1}, \ldots, \bar{s}_{i-1}, s_{i}, \bar{s}_{i+1}, \ldots, \bar{s}_{k}\right)
$$


We have:

$$
\begin{aligned}
& h \in \mathbb{R}(\mathbf{s})_{p}\left\{\begin{array}{l}
\text { for all } i \in\{1, \ldots, k\}, \\
c_{i}\left(\bar{s}^{i}\right) \text { is finite for almost all } \bar{s}^{i}
\end{array}\right. \\
& h \in \mathbb{R}(\mathbf{s})_{s p} \Longleftrightarrow\left\{\begin{array}{l}
\text { for all } i \in\{1, \ldots, k\}, \\
c_{i}\left(\bar{s}^{i}\right)=0 \text { for almost all } \bar{s}^{i}
\end{array}\right.
\end{aligned}
$$

The definition of invertibility follows from the definition used with $R$. Since $U(\mathbb{R}(\mathbf{s}))=\mathbb{R}(\mathbf{s}) \backslash\{0\}$, a matrix $A \in \mathbb{R}(\mathbf{s})^{n \times n}$ is invertible if $\operatorname{det}(A)$ is not identically zero. It follows that $\mathbb{R}(\mathbf{s})$ is in fact a field. The set $\mathbb{R}(\mathbf{s})_{p} \subset \mathbb{R}(\mathbf{s})$ is closed under addition and multiplication, but not inversion. It is therefore a subring of $\mathbb{R}(\mathbf{s})$. The invertible proper elements are precisely the set $\mathbb{R}(\mathbf{s})_{n}=U\left(\mathbb{R}(\mathbf{s})_{p}\right)$. The remaining elements are strictly proper, $\mathbb{R}(\mathbf{s})_{s p} \subset \mathbb{R}(\mathbf{s})_{p}$, and are an ideal of $\mathbb{R}(\mathbf{s})_{p}$.

Lemma 7. Suppose $G \in \mathbb{R}(\mathbf{s})_{s p}^{m \times n}$ and $K \in \mathbb{R}(\mathbf{s})_{p}^{n \times m}$. Then $(I-G K)$ is invertible, and $(I-G K)^{-1} \in \mathbb{R}(\mathbf{s})_{p}^{m \times m}$.

Proof. By Lemma 6, we have that for any $i$,

$$
\lim _{s_{i} \rightarrow \infty} \operatorname{det}(I-G K)=\operatorname{det}(I)=1
$$

for almost any assignment of the remaining variables $\left\{s_{1}, \ldots, s_{i-1}, s_{i+1}, \ldots, s_{k}\right\}$. This holds because $G$ is strictly proper and $K$ is proper. Applying Lemma 6 once more, we conclude that $\operatorname{det}(I-G K) \in \mathbb{R}(\mathbf{s})_{n}$ and so $(I-G K)$ is invertible. Furthermore, $(I-G K) \in \mathbb{R}(\mathbf{s})_{p}$. Consequently, $\operatorname{adj}(I-G K) \in \mathbb{R}(\mathbf{s})_{p}$ because $\mathbb{R}(\mathbf{s})_{p}$ is a subring. It follows that $(I-G K)^{-1} \in \mathbb{R}(\mathbf{s})_{p}$.

\subsection{Quadratic Invariance for Rationals}

Lemma 8. Suppose $G \in \mathbb{R}(\mathbf{s})_{s p}^{m \times n}$ and $K \in \mathbb{R}(\mathbf{s})_{p}^{n \times m}$. Then there exist $h_{1}, \ldots, h_{m} \in \mathbb{R}(\mathbf{s})_{n}$ such that:

$$
h(K)=\sum_{i=1}^{m} h_{i} K(G K)^{i-1}
$$

Proof. By Lemma 7, $(I-G K)$ is invertible, and so $h(K)$ is always well-defined. We may express it in terms of the classical adjoint

$h(K)=-K(I-G K)^{-1}=\frac{-1}{\operatorname{det}(I-G K)} K \operatorname{adj}(I-G K)$ and apply Lemma 2 to express the adjoint as a finite sum

$$
\begin{aligned}
& h(K)=\frac{1}{\operatorname{det}(I-G K)} \sum_{i=1}^{m} p_{i} K(I-G K)^{i-1} \\
& =\frac{1}{\operatorname{det}(I-G K)} \sum_{i=1}^{m} p_{i} \sum_{j=1}^{i}(-1)^{j-1}\left(\begin{array}{c}
i-1 \\
j-1
\end{array}\right) K(G K)^{j-1} \\
& =\sum_{j=1}^{m} \underbrace{\left[\frac{(-1)^{j-1}}{\operatorname{det}(I-G K)} \sum_{i=j}^{m}\left(\begin{array}{c}
i-1 \\
j-1
\end{array}\right) p_{i}\right]}_{h_{j}} K(G K)^{j-1}
\end{aligned}
$$

Next step is to show that $h_{j} \in \mathbb{R}(\mathbf{s})_{n}$. We will do this via Lemma 6 by showing that the limits $s_{i} \rightarrow \infty$ are finite and nonzero for almost all assignments of the remaining variables. Recall that the $p_{i}$ are defined in terms of a determinant

$$
p(x)=\operatorname{det}(I-G K-x I)=p_{0}+p_{1} x+\cdots+p_{m} x^{m}
$$

Now apply Lemma 6 . For every $i$, and any $x \in \mathbb{R}$,

$$
\lim _{s_{i} \rightarrow \infty} \operatorname{det}(I-G K-x I)=(1-x)^{m}
$$

for almost all $\bar{s}^{i}$. Equating coefficients, we find

$$
\lim _{s_{i} \rightarrow \infty} p_{i}\left(\bar{s}_{1}, \ldots, \bar{s}_{i-1}, s_{i}, \bar{s}_{i+1}, \ldots, \bar{s}_{k}\right)=(-1)^{i}\left(\begin{array}{c}
m \\
i
\end{array}\right)
$$

for almost all $\bar{s}^{i}$. Using this fact, we may now evaluate the limit of each $h_{j}$ as $s_{i} \rightarrow \infty$.

$$
\begin{aligned}
\lim _{s_{i} \rightarrow \infty} h_{j}\left(\bar{s}_{1}, \ldots, \bar{s}_{i-1}, s_{i}, \bar{s}_{i+1}, \ldots, \bar{s}_{k}\right) & \\
= & (-1)^{j-1} \sum_{i=j}^{m}\left(\begin{array}{c}
i-1 \\
j-1
\end{array}\right)\left(\begin{array}{c}
m \\
i
\end{array}\right)(-1)^{i} \\
& =-1
\end{aligned}
$$

for almost all $\bar{s}^{i}$, and we conclude that $h_{j} \in \mathbb{R}(\mathbf{s})_{n}$, as required.

Theorem 9. Suppose $G \in \mathbb{R}(\mathbf{s})_{s p}^{m \times n}$, and $S \subset \mathbb{R}(\mathbf{s})_{p}^{n \times m}$ is an $\mathbb{R}(\mathbf{s})_{p}$-module.

$$
S \text { is } Q I \text { with respect to } G \quad \Longleftrightarrow \quad h(S)=S
$$

Proof. $(\Longrightarrow)$ Choose $K \in S$. Using Lemma 8, write:

$$
h(K)=\sum_{j=1}^{m} h_{j} K(G K)^{j-1}
$$

where $h_{j} \in \mathbb{R}(\mathbf{s})_{n}$. By Lemma $4, K(G K)^{j} \in S$. Since $S$ is an $\mathbb{R}(\mathbf{s})_{p}$-module, the finite sum also belongs to $S$, and we conclude that $h(S) \subset S$. By the involutive property of $h$, it follows that $h(S)=S$.

$(\Longleftarrow)$ Suppose, conversely, that $S$ is not QI with respect to $G$. So there must exist some $K_{0} \in S$ with $K_{0} G K_{0} \notin S$. Let $r \in \mathbb{R}$ and define $K=r K_{0}$. Note that $K \in S$, since $S$ is an $\mathbb{R}(\mathbf{s})_{p}$-module. Now write:

$$
h(K)=\sum_{j=1}^{m} h_{j} K(G K)^{j-1}=\sum_{j=1}^{m} r^{j} h_{j}(r) \underbrace{K_{0}\left(G K_{0}\right)^{j-1}}_{F_{j}}
$$

Note that $h_{j}(r)$ depends on $r$. We have $F_{j} \in \mathbb{R}(\mathbf{s})_{s p}^{n \times m}$ as well. Assume that $h(K) \in S$ for every $r$. Then in particular, for any choice of nonzero $r_{1}, r_{2}, \ldots, r_{m} \in \mathbb{R}$ such that the quantities $h_{i}\left(r_{j}\right)$ are well-defined, we have:

$$
\begin{gathered}
h_{1}\left(r_{1}\right) F_{1}+\cdots+r_{1}^{m-1} h_{m}\left(r_{1}\right) F_{m} \in S \\
\vdots \\
h_{1}\left(r_{m}\right) F_{1}+\cdots+r_{m}^{m-1} h_{m}\left(r_{m}\right) F_{m} \in S
\end{gathered}
$$


Any linear combination of the sums on the left-hand side must also belong to $S$. Consider the matrix

$$
C=\left[\begin{array}{cccc}
h_{1}\left(r_{1}\right) & r_{1} h_{2}\left(r_{1}\right) & \cdots & r_{1}^{m-1} h_{m}\left(r_{1}\right) \\
h_{1}\left(r_{2}\right) & r_{2} h_{2}\left(r_{2}\right) & \cdots & r_{2}^{m-1} h_{m}\left(r_{2}\right) \\
\vdots & \vdots & \ddots & \vdots \\
h_{1}\left(r_{m}\right) & r_{m} h_{2}\left(r_{m}\right) & \cdots & r_{m}^{m-1} h_{m}\left(r_{m}\right)
\end{array}\right]
$$

By Lemma 8, it is a matrix of rational functions. In fact, $C \in \mathbb{R}(\mathbf{s})_{p}^{m \times m}$. We would like to verify that $C$ is invertible, so we apply Lemma 6 . This is straightforward since we already know the limits of the $h_{j}$ from Lemma 8. For every $i$ and for almost all $\bar{s}^{i}$,

$$
\begin{aligned}
\lim _{i \rightarrow \infty} \operatorname{det}\left(C\left(\bar{s}_{1}, \ldots, \bar{s}_{i-1}, s_{i}, \bar{s}_{i+1}, \ldots, \bar{s}_{k}\right)\right. & \\
& =(-1)^{m} \operatorname{det}\left[\begin{array}{cccc}
1 & r_{1} & \cdots & r_{1}^{m-1} \\
1 & r_{2} & \cdots & r_{2}^{m-1} \\
\vdots & \vdots & \ddots & \vdots \\
1 & r_{m} & \cdots & r_{m}^{m-1}
\end{array}\right] \\
& =(-1)^{m} \prod_{1 \leq i<j \leq m}\left(r_{j}-r_{i}\right)
\end{aligned}
$$

where we used a property of Vandermonde matrices to evaluate the determinant. As long as we choose distinct $r_{i}, \operatorname{det}(C)$ tends to a finite and nonzero limit, and so $C$ is invertible, and $C^{-1} \in \mathbb{R}(\mathbf{s})_{p}^{m \times m}$. If we treat the rows of $C^{-1}$ as coefficients, and compute the corresponding linear combinations of $(1)$, we obtain $m$ equations:

$$
F_{i} \in S \quad i=1, \ldots, m
$$

In particular, we have $F_{2} \in S$. But $F_{2}=K_{0} G K_{0} \notin S$, a contradiction. We conclude that our assumption was incorrect, so there exists some $K$ for which $h(K) \notin S$.

\subsection{IQI results}

The notion of internal quadratic invariance [4] generalizes the notion of quadratic invariance to provide a weaker sufficient condition under which the set of achievable closed-loop maps $f(P, S)$ is affine. This condition fits nicely into our algebraic framework. We begin with some preliminary definitions. If $A \in \mathbb{R}(\mathbf{s})_{p}^{m \times n}$, define

$$
\begin{aligned}
\operatorname{range} A & =\left\{A x \mid x \in \mathbb{R}(\mathbf{s})_{p}^{n}\right\} \\
\operatorname{null} A & =\left\{x \in \mathbb{R}(\mathbf{s})_{p}^{n} \mid A x=0\right\} .
\end{aligned}
$$

Both these sets are $\mathbb{R}(\mathbf{s})_{p}$-modules. Also, a matrix $W \in$ $\mathbb{R}(\mathbf{s})^{n \times n}$ is called a projector if $W^{2}=W$.

Definition 10. Let $P \in \mathbb{R}(\mathbf{s})_{s p}^{m \times n}$ and $S \subset \mathbb{R}(\mathbf{s})_{p}^{n_{2} \times m_{2}}$ be an $\mathbb{R}(\mathbf{s})_{p}$-module. Let $W_{1}$ and $W_{2}$ be any projectors such that

$$
\begin{aligned}
\text { range } W_{1} & =\text { range }\left[\begin{array}{ll}
P_{21} & P_{22}
\end{array}\right] \\
\text { null } W_{2} & =\text { null }\left[\begin{array}{l}
P_{12} \\
P_{22}
\end{array}\right]
\end{aligned}
$$

We say $S$ is internally quadratically invariant (IQI) with respect to $P$ if $W_{2} S W_{1}$ is $Q I$ with respect to $P_{22}$.

One can show that proper projectors satisfying (2) always exist. Furthermore, internal quadratic invariance does not depend on the choice of projectors $W_{i}$. In other words, internal quadratic invariance is a property of $P$ and $S$ alone. Both of these properties are proved in [4].

Lemma 11. Suppose $P \in \mathbb{R}(\mathbf{s})_{s p}^{m \times n}$ and $S \subset \mathbb{R}(\mathbf{s})_{p}^{n_{2} \times m_{2}}$ is an $\mathbb{R}(\mathbf{s})_{p}$-module. Further suppose that $S$ is internally quadratically invariant with respect to $P$, and let $W_{1}, W_{2}$ be projectors satisfying (2). Then,
i) $\left[\begin{array}{cc}I & 0 \\ 0 & W_{1}\end{array}\right] P\left[\begin{array}{cc}I & 0 \\ 0 & W_{2}\end{array}\right]=P$
ii) $h\left(W_{1} S W_{2}\right)=W_{1} h(S) W_{2}$

Proof. See Lemmata 11 and 13 in [4].

A simple consequence of Theorem 9 is that we may pre- and post-multiply by matrices $W_{1}$ and $W_{2}$, and the result will still hold.

Corollary 12. Suppose $G \in \mathbb{R}(\mathbf{s})_{s p}^{m \times n}, S \subset \mathbb{R}(\mathbf{s})_{p}^{n \times m}$ is an $\mathbb{R}(\mathbf{s})_{p}$-module, and $W_{1} \in \mathbb{R}(\mathbf{s})_{p}^{m \times m}, W_{2} \in \mathbb{R}(\mathbf{s})_{p}^{n \times n}$ are square matrices. Then,

$W_{2} S W_{1}$ is $Q I$ with respect to $G$

$$
\Longleftrightarrow \quad h\left(W_{2} S W_{1}\right)=W_{2} S W_{1}
$$

Proof. It is straightforward to verify that if $S$ is an $\mathbb{R}(\mathbf{s})_{p}$-module, then so is $W_{2} S W_{1}$. The proof follows immediately from Theorem 9 .

We can now extend Theorem 9 to the IQI case. By choosing $W_{1}$ and $W_{2}$ in a particular way, we can find a sufficient condition under which the closed-loop map is convex. This condition is weaker than the QI condition, meaning that it is more general.

Theorem 13. Let $P \in \mathbb{R}(\mathbf{s})_{s p}^{m \times n}$, and suppose $S \subset$ $\mathbb{R}(\mathbf{s})_{p}^{n_{2} \times m_{2}}$ is an $\mathbb{R}(\mathbf{s})_{p}$-module. If $S$ is IQI with respect to $P$, then

$$
P_{12} h(S) P_{21}=P_{12} S P_{21}
$$

Proof. Let $W_{1}$ and $W_{2}$ be proper projectors satisfying (2). Using Corollary 12, we have $h\left(W_{2} S W_{1}\right)=W_{2} S W_{1}$. Applying Lemma 11, this is equivalent to

$$
W_{2} h(S) W_{1}=W_{2} S W_{1}
$$

Multiply on the left by $P_{12}$ and on the right by $P_{21}$. Apply Lemma 11 again and conclude that $P_{12} W_{2}=P_{12}$ and $W_{1} P_{21}=P_{21}$. Therefore

$$
P_{12} h(S) P_{21}=P_{12} S P_{21}
$$

as required. 


\section{Examples}

In this section, we show some examples of problems that can be modeled using our algebraic framework. The purpose is to illustrate that the constraint that $S$ be an $R_{p^{-}}$ module occurs frequently and in a variety of different situations.

\subsection{Sparse Controllers}

The simplest class of systems that we can analyse are systems with rational transfer functions subject to controllers with sparsity constraints. It is clear that if every nonzero entry in the controller is required to be a proper rational function in $\mathbb{R}(s)_{p}$, the set $S$ of allowable controllers is an $\mathbb{R}(s)_{p}$-module.

\subsection{Network with Delays}

Consider a distributed system where the subsystems affect one another via delay constraints. We wish to design a decentralized controller subject to communication delay constraints between subcontrollers.

Introduce the additional variable $d$ that represents a delay of one time unit. The plant $G$ and controller $K$ are therefore rational functions in $s$ and $d$. The constraint $K \in \mathbb{R}(s, d)_{p}$ naturally guarantees that negative delays are forbidden, thus enforcing causality.

Define the Delay of a transfer function as the difference between the degree of $d$ in its denominator and numerator. For example,

$$
\operatorname{delay}\left(\frac{1}{s d+2}\right)=1 \text { and } \operatorname{delay}\left(\frac{s+d^{2}}{s^{2} d+d^{5}}\right)=3
$$

As a convention, $\operatorname{delay}(0)=\infty$. We can impose delay constraints on the controller using a set of the form

$$
S=\left\{K \in \mathbb{R}(s, d)_{p} \mid \operatorname{delay}\left(K_{i j}\right) \geq a_{i j}\right\}
$$

where $a_{i j} \geq 0$ is the minimum delay between subcontrollers $i$ and $j$. One can verify that $S$ is an $\mathbb{R}(s, d)_{p^{-}}$ module, and so we may apply Theorem 9 to derive conditions under which the problem is convex. Similar results proved using very different methods can be found in [7].

\subsection{Multidimensional Systems}

Rational functions in multiple variables with mixed properness constraints are valid in our framework. For example, suppose our transfer functions depend on two sets of variables: $R=\mathbb{R}\left(s_{1}, \ldots, s_{m}, z_{1}, \ldots, z_{n}\right)$. Further suppose that we impose a properness constraint on $s_{1}, \ldots, s_{m}$, but not on $z_{1}, \ldots, z_{n}$. This might occur, for example, if some of the variables are spatial, and it doesn't make sense to impose a properness constraint on them. This framework is used to represent spatiotemporal dynamics in a variety of important papers $[6,3,1,2]$.
The set $R$ is indeed a commutative ring, and so we may apply Theorem 5. Theorems 9 and 13 hold as well, with appropriate modifications to the notation.

\section{Conclusion}

Quadratic invariance is a property that ensures that control synthesis problems subject to structured information constraints are convex. In this paper, we show that quadratic invariance results, in rings and fields, are a consequence of fundamental algebraic properties. This fits nicely into the common practical case of rational transfer functions. In particular, we show that when the plant and controller are matrices of multidimensional transfer functions, we can easily consider sparsity, properness, delay constraints, and internal quadratic invariance.

\section{References}

[1] C. L. Beck, J. Doyle, and K. Glover. Model-reduction of multidimensional and uncertain systems. IEEE Transactions on Automatic Control, 41(10):1466-1477, 1996.

[2] R. D'Andrea and G. E. Dullerud. Distributed control design for spatially interconnected systems. IEEE Transactions on Automatic Control, 48(9):1478-1495, 2003.

[3] E. Fornasini and G. Marchesini. Doubly-indexed dynamical systems: State-space models and structural properties. Theory of Computing Systems, 12(1):59-72, 1978.

[4] L. Lessard and S. Lall. Internal quadratic invariance and decentralized control. To appear, American Control Conference, 2010.

[5] H. Matsumura and M. Reid. Commutative ring theory. Cambridge University Press, 1989.

[6] R. Roesser. A discrete state-space model for linear image processing. IEEE Transactions on Automatic Control, 20(1):1-10, 1975.

[7] M. Rotkowitz, R. Cogill, and S. Lall. Convexity of optimal control over networks with delays and arbitrary topology. International Journal of Systems, Control and Communication, 2(1):30-54, 2010.

[8] M. Rotkowitz and S. Lall. Decentralized control information structures preserved under feedback. In IEEE Conference on Decision and Control, pages 569-575, 2002.

[9] M. Rotkowitz and S. Lall. A characterization of convex problems in decentralized control. IEEE Transactions on Automatic Control, 51(2):274-286, 2006.

[10] M. Vidyasagar, H. Schneider, and B. Francis. Algebraic and topological aspects of feedback stabilization. IEEE Transactions on Automatic Control, 27(4):880894, 1982.

[11] H. S. Witsenhausen. A counterexample in stochastic optimum control. SIAM Journal on Control, 6:131, 1968. 